\title{
Characteristics of Effective Health and Safety Training Environments and Trainers in Australian Vocational Education and Training
}

\author{
Richard Skiba \\ LRES Training Management, Melbourne, Australia \\ Email address: \\ richard@skiba.com.au
}

To cite this article:

Richard Skiba. Characteristics of Effective Health and Safety Training Environments and Trainers in Australian Vocational Education and Training. International Journal of Vocational Education and Training Research. Vol. 6, No. 2, 2020, pp. 22-28.

doi: 10.11648/j.ijvetr.20200602.11

Received: June 22, 2020; Accepted: July 7, 2020; Published: July 17, 2020

\begin{abstract}
Effective health and safety training can contribute to a reduction of workplace accidents and assist employers to meet their health and safety legal obligations as prescribed by health and safety legislation. This paper considers the competency requirements for trainers of health and safety within the Australian Vocational Education and Training (VET) context from a standards perspective. Currently, there are not specific specified requirements for these trainers, but rather generic requirements that apply to all trainers in the VET sector. An interpretive qualitative method utilizing observations based on a review of a range of sources, including published research, standards documents, general internet sources and industry publications is applied such that the health and safety training practices and environments in the VET sector are explored and described. Health and safety training, in order to be effective, warrants careful attention to the training environment, the training practices implemented and the skills and knowledge of the trainer. The role of standards for health and safety training, with ANSI/ASSE Z490.1-2016 and CAN/CSA Z1001-18b provided as examples, is considered and a these standards provide a basis for a proposed standardization for health and safety training practitioners in the Australian VET system. The findings offer inclusion of a specific training and assessment unit of competency in the TAE Training and Education Training Package to address the specific requirements for safety trainers, which then be used as a benchmark in the VET sector and by employers and industry. The paper, in conclusion, posits that inconsistent training practices and trainer qualifications could be minimized by utilizing a national, consensus-based standard on the training practices in health and safety training, which can be achieved through a competency specification.
\end{abstract}

Keywords: Health and Safety, Vocational Education and Training, Trainer Competence, Training Practices, Adult Learning

\section{Introduction}

Employee training is essential to every organization's safety and health program and in most cases is a legal obligation. Any investment in such training is rewarded with reduced workplace accidents. Fundamentally, workers need to know about the hazards they face at work, as well as the methods used to reduce or control the risk of injury [1].

Vocational Education and Training (VET) plays an important role in the development of work ready individuals. One of the most significant components of a work ready individual is the ability to undertake work in their chosen industry in a safe manner. Effective training assists inexperienced workers, who, based on inexperience, tend to have higher injury and illness rates than experienced workers. Trotto [2] quantifies that employees in their first month on the job have more than three times the risk for a lost-time injury than workers who have been at their job for more than a year. Training for commencing workers, especially orientation and reinforcement, is paramount to protecting new workers according to Trotto [2].

Often training occurs prior to the worker entering their job, and this training is often provided through the Vocational Education and Training (VET) sector as individuals undertake the training required to enter the workforce, make a career change or upskill existing skills. Health and safety 
training in this regard may be embedded within a unit or may be specific health and safety training and examples of both approaches are common [3]. Billorou and Sandoya [4] recognise that the inclusion of health and safety training in vocational training programs is commonplace. They note, however, that most programs "use a generic approach that focuses on the transmission of conceptual and informative content rather than a practical approach that allows students to develop prevention practices alongside functional job-related skills" [4].

Effective health and safety training offers a number of benefits including meeting legal obligations as prescribed by health and safety legislation, reduced workplace accidents and incidents based on improved hazard identification and application of controls and increased efficiency with workers better being able to focus on work tasks rather than trying to manage unsafe work conditions. Given the benefits of effective health and safety training, effectiveness requires definition and, effective implementation within a vocational education and training setting requires equal consideration. The definition is best considered by characterizing a specific unit of competency focusing on the skills and knowledge required to train learners in safe work practices.

\section{Method}

A qualitative research is utilized for this study, relying on non-numeric data, primarily observations in this case, in contrast to quantitative research which employs numeric data such as scores and metrics [5]. This study takes a social constructivist approach where social constructivism is an interpretive framework, as outlined by Creswell [6]. Throughout this research, the researcher seeks to develop particular meanings that correspond to their experience based on observations obtained by reviewing the views of others. In social constructivism human, interests are important for research purposes and knowledge is constructed through social interaction and discourse, and as such knowledge is shared rather than an individual experience by means of collaboration. Creswell further notes that meanings are formed through interaction with others. The aim of constructivist research applied here is to understand a particular situation or phenomena, that of effective training and assessment in health and safety training practices.

The approach recognises that knowledge is a human product and is socially and culturally constructed. Social constructivism emphasizes the importance of culture and context in the process of knowledge construction and accumulation. The method applied here is interpretive in that the researcher uses interpretation to make sense of a social situation rather than engaging in a hypothesis testing process. This method lends itself well to the phenomenon under consideration within this study and is appropriate for studying context-specific, unique, or idiosyncratic events or processes [5]. Observations are based on a review of a range of sources, including published research, standards documents, general internet sources and industry publications and these are analyzed for the purposes of exploration and description. Munkvold and Bygstad [7] note that interpretive research aims for analytic generalization, in the sense of producing results that have implications beyond the studied research setting, as is the objective of this study.

\section{Discussion}

\subsection{An Effective Safety Training Environment}

Training is not likely to be of benefit to workers do not understand it, if they are unmotivated, if they have poor work attitudes or they are unable to put the learning into practice. The training environment must be established in a way that learners are engaged, motivated and inspired.

Considering particularly workplace-based training, Townsell [8] posits that assessing and analyzing employee training needs prior to delivering the program can strengthen the impact of the program. The key being, identified by Townsell, to focus the training specifically to the individual and their specific workplace environment. Focused training includes specificity, relevance and conciseness. In order to do so, content must be relevant to the job skills that will be performed and relevant to the jurisdiction in which the work will take place. This targeted approach provides for analyzing the specific hazards that the learner will encounter and, in turn, will learn to manage. Training needs, Townsell [8] suggests, can be determined through conducting hazard analysis, utilization of a training needs assessment questionnaire and through a range of other methods including "interviews, job observation (which is also a component of a job/task hazard analysis), focus groups and analyzing accident and near miss data and statistics". Cekada [9] advises that a training needs assessment is used to determine whether training is the right solution to a workplace problem. The process can and should be applied to health and safety training activities prior to implementing the training.

Undertaking hazard analysis allows the trainer to take the learner's destination environment and break the job role into its applicable hazards. Once these are determined, proactive measures can be identified and the safety training can be framed around these. Townsell [8] outlines that there are a range of tools that can be used to undertake the hazard analysis including Fault Tree Analysis, Job/Task Hazard Analysis and the Hazard and Operability Study.

Some guidance with regard to the delivery of safety training is provided by 'ANSI/ASSE Z490.1-2016 - Safety, Health, and Environmental Training' (formerly ANSI/ASSE Z490.1-2016) as produced by the American Society of Safety Engineers and as the current release of the standard. The standard ANSI/ASSE Z490.1-2016 provides employers with the means to carry out this training, touching upon all facets of the training process and establishes voluntary criteria for safety, health, and environmental training programs, including development, delivery, evaluation and program management. The standard is designed to apply to all safety, health, or environmental training, whether separate or a 
portion of other training. A further key element of the standard is that it offers performance-oriented guidance on all the essential elements of an effective training process.

Dunn [10] recognises that part of the standard addresses the importance of conducting a needs assessment when developing training, supporting the use of a training needs analysis as advocated by Townsell [8]. While the standard stresses the importance of understanding the audience and requires that learning objectives detail the target audience, it does not include a step-by-step explanation of how to perform an audience analysis [10]. Audience analysis can be categorized as:

"Audience analysis is the process of fully analyzing the audience, then using that analysis to craft information that meets the audience's needs. The language, graphics and even the chosen medium (e.g., print, video, web) are all designed to cater to the designated audience. The goal is to make sure the audience has everything it needs to process, fully understand and use the information". [10, p 31].

The ANSI Z490.1 standard condones, as it first step, conducting a needs assessment to ensure that training is the correct response to the business problem, or the hazard exposure presented [1]. Where training is an appropriate response, the assessment should continue and by clearly defining the audience to be trained, the course objectives, the content to be covered during the course, the specific training methods to be used, how learning will be measured, and how the overall effectiveness of the training will be assessed post-training.

As an example of another training standard related to health and safety training, the Canadian Standards Association (CSA) has developed the CAN/CSA Z1001-18b Occupational health and safety training, superseding the previous edition published in 2013, that provides the essentials for managing an Occupational Health and Safety (OHS) training program. The Standard specifies requirements for management and administration of OHS training, identification of OHS training needs, and outlines the development, implementation, and maintenance of an OHS training program and courses. It also specifies the requirements for the design and delivery of OHS training courses. This standard specifies that trainers should receive training in how to instruct and in their practices: plan the session beforehand; break the job down into steps; have training aids available; explain what is to be done; describe all the hazards and protective measures; demonstrate each step, stress key points, and answer any questions; and, have the learners carry out each step, correct errors, and compliment good performance [11].

\subsection{An Effective Safety Trainer}

Townsell [12] provides an overview of the general characteristics for those providing safety training. The first of these is that trainers are required to be subject matter experts. Any trainer engaging in health and safety training must be competent in the area themselves. They must not only have a thorough knowledge of the topics being taught, they should also have relevant experience. A trainer in the Australian Vocational Education and Training system, must be 'vocationally competent' in any areas they are training where 'vocational competency' id defined to include "broad industry knowledge and experience, usually combined with a relevant industry qualification" [13]. These trainers should also have a desire to teach whereby "effective trainers have a desire to impart their knowledge to others" [12]. Another identified characteristic is that of having a positive, helpful and cooperative attitude which is required to engage students during the training activity. This characteristic is tied to strong leadership abilities that are required to direct learners to the required objective. A professional attitude and provision of exemplary behaviour are also contributors to effective health and safety training.

The ANSI/ASSE Z490.1 standard defines that safety, health, and environmental trainers should be able to demonstrate an appropriate level of technical knowledge, skills, or abilities in the subjects they teach and be able to demonstrate adequate competency in delivery techniques and methods appropriate to adult learning [1]. They should also maintain competency by participating in continuing education, development programs, or experience related to their subject matter. On application of adult learning principles, the trainer should be able to apply these as appropriate to their target audience and adult learning principles should also be reflected in the learning objectives.

An effective health and safety trainer should possess specific knowledge and skills including [14]:

a) leadership skills.

b) communication skills.

c) health and safety principles.

d) techniques of safety and health management.

e) accident analysis techniques and prevention strategies.

f) training, instruction, coaching and problem - solving skills relevant to safety and health.

g) understanding of the hazards and risks related to specific job roles.

h) detailed knowledge of the safety and health arrangements relevant to an individual's job.

i) knowledge of relevant legislation and appropriate methods of control, including risk assessment.

j) knowledge of the organization's planning, measuring, reviewing and auditing arrangements.

k) awareness of the financial and economic benefits of good safety and health performance.

\subsection{An Effective Safety Training Method}

Sharp [15] notes that learners react to and absorb information differently. Sharp suggests that the Kolb four stage learning cycle can be a useful approach to safety training, noting that this "approach actively engages employees in the learning process and provides them with the experience that they're most likely to learn from, which in turn encourages more reflection and continuous learning" [15]. McLeod [16] explains that Kolb states that learning involves the acquisition of abstract concepts that can be 
applied flexibly in a range of situations and that the development of new concepts is provided by new experiences.

The four stage learning cycle is characterized by: Concrete Experience, Reflective Observation; Abstract Conceptualization; and, Active Experimentation. The concrete experience stage includes learning from doing, where a new experience or situation is encountered, or based on a reinterpretation of existing experience. Learners learn from new experiences in a training environment through participation and engagement and a 'hands-on' approach is effective in this regard. Reflective observation suggests that learners can learn by reflecting on past experience. Abstract conceptualization relates to formation of new ideas based on past experience. At this stage of learning, reflection gives rise to a new idea, or a modification of an existing abstract concept and the individual, in turn, learns from their experience. The final stage, active experimentation, includes taking the ideas developed in the abstract conceptualization stage and testing them out.

Kolb's process then can be seen as a means of effective learning whereby through a concrete experience followed by observation of and reflection on that experience, a learner moves to the formation of abstract concepts and generalizations which are then used to test a hypothesis in future situations, resulting in new experiences [16]. Kolb [17] presents learning as an integrated process with each stage being mutually supportive of and feeding into the next. It is a process whereby possible to enter the cycle at any stage and follow it through its logical sequence. This approach actively engages learners in the learning process and provides them with the experience that they're most likely to learn from, ultimately encouraging more reflection and continuous learning. Stuart [18] concurs noting that Kolb's theoretical model of experiential learning has particular relevance for disciplines that employ more active or experience-based learning and teaching approaches, such as is most commonly the case in vocational education and training.

Learner learning styles are another consideration in training effectiveness. In this regard Kolb has described four basic learning styles: Accommodative, Assimilative, Divergent and Convergent [17]. Stuart [18] describes Accommodating Learners as those who have the ability to learn primarily from "hands-on" experience. Converging Learners excel at finding practical uses for ideas and theories. Assimilative Learners prefer readings, lectures, exploring analytical models, and having time to think things through. Diverging Learners are learners with a preference to work in groups, listening with an open mind and receiving personalised feedback. An awareness of a learner's preferred learning style by a Trainer can assist the formation of a training approach to maximize that learner's learning and the subsequent application of that learning.

Pollock [1] discusses the barriers to safety training and proposes some means by which these can be removed. Pollock notes that "a portion of safety training can be categorized as informational and may not convey any responsibility or authority to take action" and "the people in the class usually do not report to the trainer, so there is little if any call to action that will translate to the work environment". These are two issues that can potentially create a barrier to training and need to be addressed by both the trainer and the training environment. Information must be contextualized for the learners and made relevant to their work environments. Möckel, Brenker and Strohschneider [19] suggest that accident analysis, specific to a work environment or situation can be a useful tool in effective information presentation.

Pollock [1] further identifies that cultural barriers are common and may stand in the way of effective health and safety training and subsequent application of the training. Pollock suggests that these can be identified through perception surveys, or during conversations with the workers and their supervisors, and as such, health and safety training requires ongoing industry engagement by the trainer such that they are fully aware of specific industry and employer culture. They can then be factored into the training program and approach. Pollock posits that an effective way to overcome cultural barriers is to include managers and supervisors in the training with their employees as far as is practicable. Once aware and trained the managers are better able to discuss safety, set priorities and drive lasting change.

\subsection{An Australian Vocational Education and Training Perspective}

In an Australian context, the VET system utilizes units of competency which are specifications of knowledge and skill, and the application of that knowledge and skill, to the standard of performance expected in the workplace. They essentially provide a description of the skills and knowledge required to perform effectively to a specific standard in a particular workplace role or function. In a competency-based training (CBT) program, people gain the skills and knowledge that they need to be able to perform their work. CBT programs are based on what people at work are expected to do and the standard of performance expected in the workplace.

Health and safety can be included in training packages either as dedicated units of competency or as embedded components within units of competency. In the case of a dedicated unit, which focuses on a skill function and normally encompasses an industry [3]. The skill is usually the name of the unit, for example 'communicate in the workplace', or 'apply occupational health and safety'. An embedded unit, outlines Gibb, combines the skill with a technical or work function, such as 'negotiate a contract' or 'plan and manage conferences'. Gibb [3] also notes that training packages in general have most often opted for this approach.

Although not specifically recognised as a core skill in the Australian Core Skills Framework (ACSF), health and safety is recognised as a generic skill and written into many units of competency, particularly at Certificate I to IV levels. Dawe [20], and confirmed by Gibb [3], found that communicating, 
working in teams and following occupational health and safety procedures are all generic skills in training packages they investigated.

Health and safety is often embedded as a knowledge item with word to the effect of "relevant safety, work health and safety (WHS)/occupational health and safety (OHS) and environmental procedures and regulations "and/or performance evidence to the effect of "applying relevant rail safety and workplace procedures“. Trainers however are not required to specifically develop the skills and knowledge to deliver and assess this aspect of competency effectively.

The 'Standards for Registered Training Organisations (RTOs) 2015' specify the minimum requirements for trainers and assessors. The requirements specify that training and assessment may only be delivered by trainers and assessors who hold the required credentials, hold vocational competencies at least to the level being delivered and assessed, have current industry skills directly relevant to the training and assessment being provided, have current knowledge and skills in vocational training and learning that informs their training and assessment and undertake relevant professional development [21].

From 1 July 2019, only a person who holds one of the following credentials can deliver training and assessment: TAE40116 Certificate IV in Training and Assessment; TAE40110 Certificate IV in Training and Assessment plus the following units - either TAELLN411 or TAELLN401A, and either TAEASS502 or TAEASS502A or TAEASS502B; or, a diploma or higher level qualification in adult education. Assessment can be undertaken by a person who holds one of the above credentials or the TAESS00011 Assessor Skill Set or both the TAESS00001 Assessor Skill Set and TAEASS502 Design and develop assessment tools. There are no specific standards specified for those in the VET sector to teach health and safety. The standards do specify requirements for vocational competence, skills currency and ongoing professional development, however these are not prescribed and as such there is no base competency standard for a trainer specifically related delivery of health and safety training. As such, a nationally consistent approach based on best practice can not be applied.

For the TAE40116 Certificate IV in Training and Assessment qualification, the packing rules state [22]:

"Total number of units $=10$

9 core units plus

1 elective unit

The elective unit may be:

a) from the elective list below.

b) from any currently endorsed Training Package or accredited course at Certificate IV or above.

The elective unit chosen must be relevant to the work outcome and meet local industry needs.

Core Units

TAEASS401 Plan assessment activities and processes.

TAEASS402 Assess competence.

TAEASS403 Participate in assessment validation.

TAEASS502 Design and develop assessment tools.
TAEDEL401 Plan, organise and deliver group-based learning.

TAEDEL402 Plan, organise and facilitate learning in the workplace.

TAEDES401 Design and develop learning programs.

TAEDES402 Use training packages and accredited courses to meet client needs.

TAELLN411 Address adult language, literacy and numeracy skills.

Elective Units

TAEASS301 Contribute to assessment.

TAEDEL301 Provide work skill instruction.

TAEDEL403 Coordinate and facilitate distance-based learning.

TAEDEL404 Mentor in the workplace.

TAEDEL501 Facilitate e-learning.

TAELLN412 Access resources and support to address foundation skills.

TAELLN413 Integrate foundation skills into vocational training delivery.

TAETAS401 Maintain training and assessment information.

TAEXDB401 Plan and implement individual support plans for learners with disability.

BSBAUD402 Participate in a quality audit.

BSBCMM401 Make a presentation.

BSBLED401 Develop teams and individuals.

BSBMKG413 Promote products and services.

BSBREL402 Build client relationships and business networks.

BSBRES401 Analyse and present research information".

As such, the baseline training and assessment qualification for trainers and assessors in the Australian VET system, does not include a unit that can focuses on training health and safety, in the way that it does adult language, literacy and numeracy skills or e-learning for example. Given the lack of a national standard for training health and safety, such as ANSI/ASSE Z490.1-2016 or CAN/CSA Z1001-18b, and the structure of Vocational Education and Training in Australia, a defined competency standard for trainers and training in this area would provide significant benefit to industry through increased understanding and application by learners in their respective workplaces. The increased understanding and application leading to an improved safety culture and, in turn, reduced workplace accident and incidents. Likewise, a benchmark would be established for the various practitioners and stakeholders in the VET system with a major benefit being a consistent best practice approach being implemented nationally, such as is the purpose of both the TAE40116 Certificate IV in Training and Assessment qualification and the Standards for Registered Training Organisations (RTOs) 2015.

A training and assessment competency standard related to training health and safety would be well suited to be included in the TAE Training and Education Training Package which could then potentially be offered as an elective in the TAE40116 Certificate IV in Training and Assessment 
qualification or as a stand alone unit delivered to specialist trainers, particularly those delivering dedicated health and safety units.

The unit should specify, as advocated by Billorou and Sandoya [4], the teaching strategy and the formative and summative assessment requirements, such that the practices and knowledge linked to safe and healthy work are considered and assessed at the same level as the learning of technical skills. It should also specify competency requirements for the development and application of specific training and support materials relating to health and safety, use of teaching practices effective to ensure that trainers pay ongoing attention to the subject and establishing learning environments, offering the best conditions conducive to health and safety training and learning.

\section{Conclusion}

Health and safety training is important and effective in reducing workplace incidents as it educates employees on proper workplace procedures, practices, and behaviour. Health and safety training should exist beyond the training of generic aspects in order to effectively facilitate the handling of new and uncertain situations. The training can most effectively be delivered by individuals who have the specialist skills in both training practices and health and safety systems. Diverse training practices and inconsistent trainer qualifications necessitate a need for a national, consensus-based standard on the training practices in health and safety training, which can be achieved through a competency specification. By standardizing training, employers may gain confidence that there are common standards in place to provide all VET trained personnel attending their sites with the knowledge and skills required to reduce risk, stay safe and leave injury-free.

\section{References}

[1] Pollock, R. A. (2010, January). Effective Safety Training: Tips for Engaging the Adult Learner. In ASSE Professional Development Conference and Exposition. American Society of Safety Engineers.

[2] Trotto, S. (2016). New workers, higher risk. Retrieved from https://www.safetyandhealthmagazine.com/articles/14053-ne w-workers-higher-risk.

[3] Gibb, J. (2004). Generic Skills in Vocational Education and Training. Australian National Training Authority, Adelaide SA.

[4] Billorou, N. \& Sandoya, J. (2019). Guide for the mainstreaming of Occupational Safety and Health (OSH) in vocational training programmes. Montevideo: ILO/Cinterfo.

[5] Lumen Learning. (2020). Research Methods for the Social Sciences: Chapter 12 Interpretive Research. Retrieved from https://courses.lumenlearning.com/atd-herkimer-researchmeth odsforsocialscience/chapter/chapter-12-interpretive-research/.

[6] Creswell, J. W. (2013). Qualitative inquiry \& research design: choosing among the five approaches. Thousand Oaks, CA:
Sage Publications, Inc.

[7] Munkvold, B. E. and Bygstad, B.: (2016). The Land of Confusion - Clearing up some common misunderstandings of interpretive research. Paper presented at NOKOBIT 2016, Bergen, 28-30 Nov. NOKOBIT, 24 (1), Bibsys Open Journal Systems.

[8] Townsell, J. (2012a). Who Needs This Training? (Hint: Not Everyone). Retrieved from https://www.ehstoday.com/ehs-outloud-blog/article/21915243/ who-needs-this-training-hint-not-everyone.

[9] Cekada, T. L. (2010). Training Needs Assessment: Understanding what employees need to know. Professional Safety, March, 28-33.

[10] Dunn, C. K. (2015). Audience Analysis: Taking Employees from Awareness to Understanding. Professional Safety, November, 30-34.

[11] Canadian Centre for Occupational Health and Safety. (2020). Basic OH\&S Program Elements. Retrieved from https://www.ccohs.ca/oshanswers/hsprograms/basic.html.

[12] Townsell, J. (2012b). The Characteristics of an Effective EHS Trainer Retrieved from https://www.ehstoday.com/ehs-outloud-blog/article/21918511/ the-characteristics-of-an-effective-ehs-trainer.

[13] Australian Skills Quality Authority. (2020a). How can I demonstrate vocational competency? Retrieved from https://www.asqa.gov.au/faqs/how-can-i-demonstrate-vocation al-competency.

[14] Health and Safety Authority. (2020). Safety and Health Management System. Retrieved from https://www.hsa.ie/eng/Topics/Managing_Health_and_Safety/ Safety_and_Health_Management_Systems/.

[15] Sharp, C. (2018). Effective training tips that employees will actually care about. Retrieved from https://www.turbinehq.com/blog/tips-for-effective-training.

[16] McLeod, S. (2017). Kolb's Learning Styles and Experiential Learning Cycle. Retrieved from https://www.simplypsychology.org/learning-kolb.html.

[17] Kolb, D. A. (1984). Experiential learning: Experience as the source of learning and development (Vol. 1). Englewood Cliffs, NJ: Prentice-Hall.

[18] Stuart, A. (2014). A blended learning approach to safety training: Student experiences of safe work practices and safety culture. Safety Science 62, 409-417. https://doi.org/10.1016/j.ssci.2013.10.005.

[19] Möckel, S., Brenker, M., \& Strohschneider, S. (2014). Enhancing Safety through Generic Competencies. TransNav, 8 (1). https://doi.org/10.12716/1001.08.01.11.

[20] Dawe, S. (2001). Do training packages focus sufficiently on generic skills? Paper presented at the Knowledge demands for the new economy, 9th Annual International Conference on Post-compulsory Education and Training, 3-5 December, Surfers Paradise.

[21] Australian Skills Quality Authority. (2020b). Meeting trainer and assessor requirements. Retrieved from https://www.asqa.gov.au/resources/fact-sheets/meeting-trainer -and-assessor-requirements. 
[22] Department of Employment, Skills, Small and Family Business. (2020). Qualification Details: TAE40116 - Certificate IV in Training and Assessment (Release 2). Retrieved from https://training.gov.au/Training/Details/TAE40116. 